\title{
Periodontal disease and obesity in an Indian population
}

\author{
Giri DK, ${ }^{1}$ Kundapur PP, ${ }^{2 *}$ Bhat $G S,{ }^{2}$ Bhat $K M,{ }^{2}$ Guddattu $V^{3}$ \\ ${ }^{1}$ Department of Periodontics, B.P Koirala Institute of Health Sciences, Dharan, Nepal, \\ ${ }^{2}$ Department of Periodontology, Manipal College of Dental Sciences, Manipal, \\ Manipal University, Karnataka, India, ${ }^{3}$ Department of Statistics, Manipal University, Manipal,
}

*Corresponding Author:

Pratibha P Kundapur

MDS, Additional Professor,

E-mail: bg_pratibha@yahoo.co.in

\section{Citation}

Giri DK, Kundapur PP, Bhat GS, Bhat KM, Guddattu V. Periodontal disease and obesity in an Indian population. Nepal Journal of Medical Sciences 2013;2(2):144-9.

\begin{abstract}
Background: Associations between obesity and several chronic inflammatory diseases are emerging. Research from some parts of the world has also indicated that a relationship between periodontal disease and obesity is possible. The aim of the present study was to determine the association of obesity with periodontal disease in a semi urban Indian population.
\end{abstract}

Methods: Body Mass Index (BMI) was determined for 323 patients visiting the department of Periodontics. Other demographic variables such as age, gender, stress, educational background and smoking were also considered. Clinical examination of the periodontal health was assessed using the Plaque index (PI), the Gingival index (GI) and the Community Periodontal Index (CPI).

Results: No association was obtained between BMI and periodontitis. Other demographic variables were also not significantly related. Lower gingival index scores, were significantly associated with better periodontal health.

Conclusion: Good oral hygiene and normal body weight can reduce the overall inflammatory burden, thereby reducing the risk for development of periodontal disease.

Keywords: Body Mass Index; obesity; periodontal disease

\section{Background:}

Obesity is becoming an alarming problem worldwide. In some developing countries, obesity will soon surpass the prevailing problem of malnutrition. The related health consequences such as hypertension, diabetes mellitus, cardiovascular disease, have also shown an increase with the increasing prevalence of obesity. ${ }^{1,2}$ Recently, obesity has also been suggested as a risk factor for periodontitis. ${ }^{3,4}$ Periodontitis is a multi-factorial disease characterized by an ongoing infection and inflammation in the gingiva and the destruction of tissue attachment and bone surrounding teeth. Several studies have described an epidemiological association between periodontitis and obesity, adding another dimension to the etiology of periodontal disease. ${ }^{5}$ According to Saito et al, Japanese obese subjects were more likely to have periodontal disease. ${ }^{6}$

Findings also support the association between cardiovascular disease and periodontitis. ${ }^{7}$ Diabetes is another well recognized 
risk factor for periodontitis. Periodontal condition worsens with diabetes and conversely uncontrolled periodontitis also affects glycemic control. ${ }^{8}$ Hence, considering the relation between periodontal disease, cardiovascular disease and diabetes, it is important to determine the association between periodontal disease and obesity. Literature in this regard has been lacking in the Indian population, which is a population considered to be at high risk for non-communicable diseases, and hence the need for the present investigation.

The aim of the present study was to determine the association of obesity with periodontal disease, using Body Mass Index (BMI) as an indicator of obesity. An attempt was also made to determine the influence of demographic factors such as age, gender, stress, educational background and smoking on the development of periodontitis, in a semi urban Indian population of a university township of Manipal.

\section{Methods:}

The present cross sectional study was conducted in the Periodontics department of Manipal College of Dental Sciences, Manipal University. The data for the present research were collected by interviews and clinical oral health examination. The patients were given information regarding their participation in the study and all the individuals gave consent in writing. Ethical clearance for the study was obtained from the Kasturba Hospital Ethical Committee prior to the commencement of the study.

A convenience sample of 323 subjects was selected from among those who visited the department of Periodontics during the period January to July 2011. Patients with significant medical history, receiving anti-inflammatory medications or nutritional supplements, undergoing weight loss therapy, pregnant and lactating women and those receiving periodontal therapy in the preceding six months were excluded from the study. Only subjects above 18 years were considered for inclusion. The patients were screened for body weight, height, age, gender, stress, educational background and smoking. Patients were divided into 3 groups according to age: $<30$ years, $30-45$ years, $>45$ years. Gender was categorized as male and female. The socioeconomic status was divided into lower, middle and higher strata based on their occupation. Unskilled workers and unemployed individuals were grouped in the lower strata; those in clerical jobs, skilled and semi-skilled workers, farmers, shop owners were included in the middle strata and those employed in salaried professions such as professors, doctors, teachers, corporates, bank officers etc. were included in the higher economic strata. Similarly, educational background was classified as basic if subjects were illiterate or with primary and middle school level of education, secondary education if they had completed high school or diploma qualifications and higher education if subjects had professional or honors, graduate and post graduate degrees. Smoking habit was categorized as yes/no. Stress was determined on the basis of answer option yes/no.

A single examiner was trained to conduct the interview and record the clinical data. All participants underwent a clinical periodontal examination. The oral hygiene of six selected teeth was assessed using the Plaque Index (PI) and the Gingival Index (GI). ${ }^{9-10}$ The six teeth chosen were the maxillary right first molar, the maxillary right central incisor, the maxillary left first premolar, the mandibular left first molar, the mandibular left lateral incisor and mandibular right first premolar. The scoring ranges for the Plaque Index were Good (0-0.9), Fair (1.0-1.9), Poor (2.0-3.0). The Gingival Index ranged from 0-1.0 for mild gingivitis, 1.12.0 for moderate gingivitis and 2.1-3.0 for severe gingivitis. The Community Periodontal Index was used to assess the periodontal status with a CPI probe. ${ }^{11}$ The teeth examined were $16,17,11,26,27,31,36,37$, 46, and 47. Subjects were considered as having 'Periodontitis', if the CPI score was ( 3 or 4 ) and 'No - periodontitis' if the CPI score was $(0,1$, and 2$)$.

BMI was used to indicate overall obesity $\left(\mathrm{kg} / \mathrm{m}^{2}\right)$. It was calculated using each participant's weight in kilograms divided by the square of height in meters. As recommended by WHO, the international classification for adults (WHO) was followed: patients were categorized as underweight, normal weight, overweight and obesity are $<18.5 \mathrm{~kg} / \mathrm{m}^{2}$, 18.50 - $24.99 \mathrm{~kg} / \mathrm{m}^{2}, 25.00-29.99 \mathrm{~kg} / \mathrm{m}^{2}, \geq 30.00 \mathrm{~kg} / \mathrm{m}^{2}$ respectively. ${ }^{12}$

Statistical analysis:

Data was analyzed using SPSS version 16 and Chi-Square test was used to determine the association of BMI with periodontitis.

\section{Results:}

The present study comprised a total of 323 participants including 216 males and 107 females. The prevalence of periodontal disease according to socio-demographic variables, oral health parameters and clinical characteristics are shown in Table 1.

The study included 112 obese and 211 non-obese subjects. In the obese group, $54(48.2 \%)$ had periodontitis and in the non-obese group $90(42.7 \%)$ were observed to have 
periodontal diseases $(\mathrm{p}=0.339)$.

Table 1: Descriptive variables

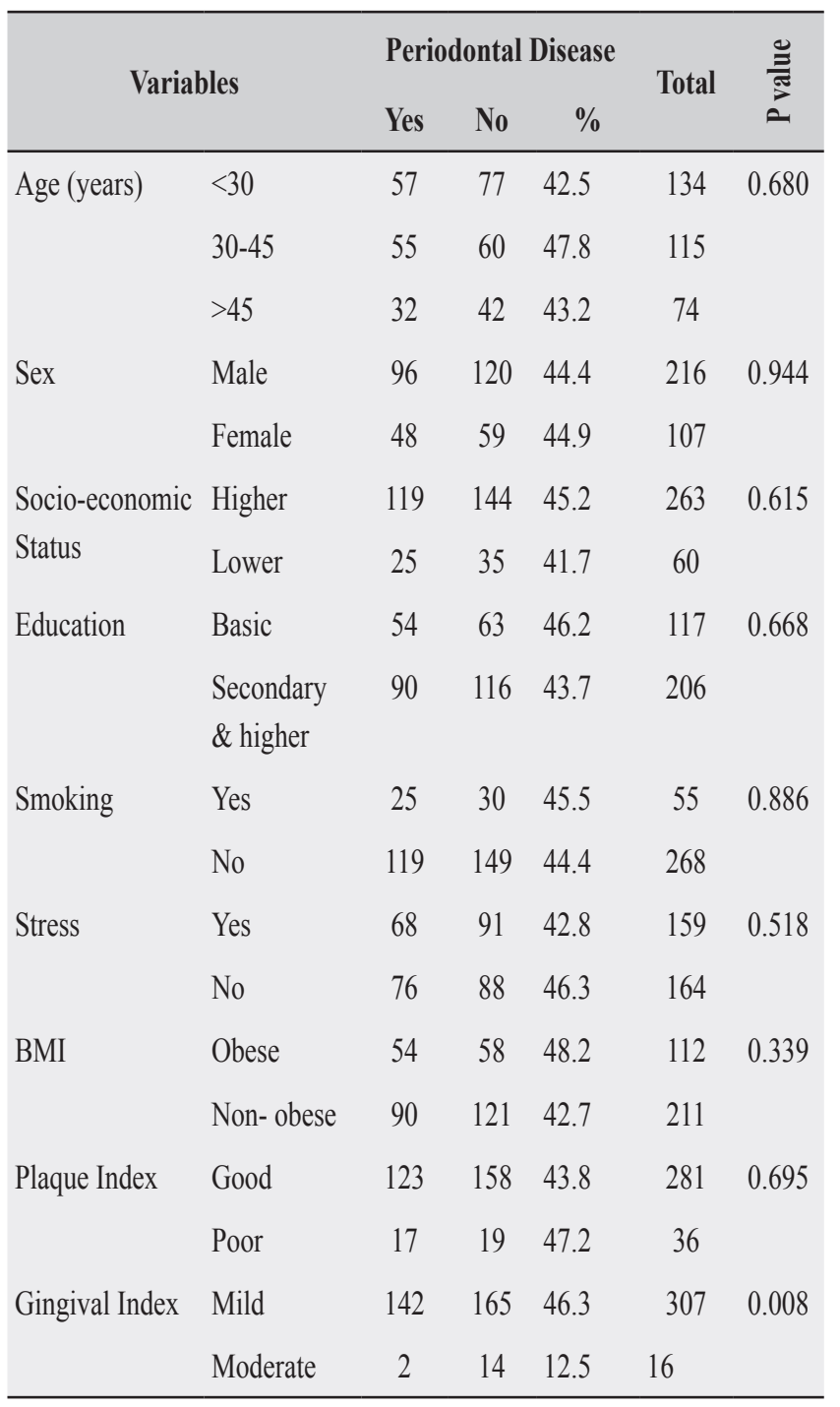

Age group $<30$ years had 134 subjects (41.48\%), age group 30 - 45 years had 115 subjects (35.61\%) and age group of $>$ 45 years had 74 subjects $(22.91 \%)$. In the age group $<30$, $42.5 \%$ of the subjects had periodontitis. In the age group 30 $45,47.8 \%$ of the subjects had periodontitis and in the group $>45,43.2 \%$ of the subjects had periodontitis $(\mathrm{p}=0.680)$.

There were 216 males and 107 females in the study. Ninety six $(44.4 \%)$ out of 216 males had periodontitis and 48 $(44.9 \%)$ out of 107 females had periodontitis $(\mathrm{p}=0.944)$.

In the higher socioeconomic strata $119(45.2 \%)$ out of 263 had periodontal disease compared to $25(41.7 \%)$ out of 60 in the lower socioeconomic strata group $(\mathrm{p}=0.615)$.

In the basic education group, 54 of $117(46.2 \%)$ subjects showed periodontitis compared to 90 (43.7\%) out of 206 subjects in the secondary and higher education group ( $p$ value 0.668$)$.
There were 55 smokers and 268 non-smokers in the study. In the smoking group, 25 (45.5\%) had periodontitis and $119(44.4 \%)$ had periodontitis in the no-smoking group ( $p$ $=0.886)$.

$68(42.8 \%)$ of the subjects out of 159 having stress had periodontitis when compared with $76(46.3 \%)$ subjects out of 164 not having stress.

It was observed that the Plaque index was not associated with periodontal disease $(\mathrm{p}=0.695)$ as lower plaque scores were seen in majority of the subjects examined.

There were 16 subjects with moderate to severe gingivitis whereas mild gingival inflammation was evident in 307 subjects. Majority of the subjects with lower gingival index scores (mild gingivitis) were periodontally healthy. This value was statistically significant $(\mathrm{p}=0.008)$.

\section{Discussion:}

Out of the total sample of 323 subjects, 144 were observed to have periodontitis and 123 of these were systemically healthy and 21 subjects were not healthy.

In the present study, subjects categorized as obese $(\geq 30 \mathrm{~kg}$ $/ \mathrm{m}^{2}$ ) were observed to have no association with periodontal disease $(p=0.339)$. Our findings are consistent with the study done by Kim EJ et al (2010) ${ }^{13}$ where he found no association between BMI and periodontitis but a significant association between abdominal obesity and periodontitis was found. Y Lostalo et al (2008) ${ }^{14}$ detected a weak exposure association of BMI with deepened periodontal pocket.

Our study utilized the BMI classification as given by WHO to determine obesity. ${ }^{12}$ However, it is now being suggested that a distinctive classification may be required for the Asian population ${ }^{15}$ because of their susceptibility to obesity related medical conditions, where BMI $\geq 25 \mathrm{~kg} / \mathrm{m}^{2}$ is considered as obese. The modified classification system suggests that $\mathrm{BMI}<18.5 \mathrm{~kg} / \mathrm{m}^{2}$ indicates underweight, $18.5-22.9 \mathrm{~kg} / \mathrm{m}^{2}$ indicates normal weight, $23-24.9 \mathrm{~kg} / \mathrm{m}^{2}$ may be considered as overweight, and $\geq 25 \mathrm{~kg} / \mathrm{m}^{2}$ are obese. ${ }^{15}$ It is possible that our study may have overlooked a large number of obese subjects by not considering this modified classification.

Further, the present study utilized BMI as a measure of obesity. It would be more reliable to use waist circumference or waist - hip ratio ${ }^{12}$ as there is a tendency for the people of Asian origin to accumulate fat in the waist-hip region.

The study showed no association between gender and periodontitis. This is contrary to a report by Dalla Vecchia CF et al (2005), ${ }^{3}$ which suggests that the topographic 
distribution of body fat in females might predispose them to periodontal disease.

Age did not show any association with periodontitis. These findings are consistent with those of Papapanou PN et al $(1991)^{16}$ where he stated that, "age related changes in the periodontium may not inevitably be manifested as loss of probing attachment or alveolar bone" and the "increase in prevalence and severity of periodontal disease with age must be the result of a prolonged exposure of the periodontal tissue to plaque rather than the consequence of age".

Abdellalif and Burt (1997) ${ }^{17}$ reported that age is not a determinant of periodontal disease when high levels of oral hygiene are maintained. In the present study, good oral hygiene was observed in 281 subjects of whom 123 had periodontitis whereas 158 subjects had no periodontitis. Hence, there was no significant influence of plaque scores on the development of periodontal disease.

The Gingival index was taken to assess the role of subgingival plaque and plaque retained in inaccessible areas over a prolonged period of time. It was observed that the scores of Gingival Index corroborated with the plaque scores with most patients exhibiting mild gingival inflammation. A majority of the patients had relatively healthy gingivae with markedly lower gingival index scores.

Out of 144 patients with periodontitis, only 25 were noted to be smokers. We did not consider information about the duration and number of cigarettes smoked. Hence, no influence of smoking on periodontitis was evident in the present study. The study done by Xu F et al $(2007)^{18}$ showed an inverse relationship of smoking in obese individuals.

Smoking may be a reflection of underlying stress. Stress, however, was not significantly associated with periodontitis in the present study. This was also consistent with the fact that fewer smokers were seen in the study group.

It was also observed that a majority of the subjects who were examined belonged to a higher socio economic stratum with higher levels of education, possessing good oral hygiene and minimal gingival inflammation. As most of the subjects belonged to a university population, there was apparently a good degree of motivation towards dental care with access to better health care facilities.

\section{Conclusion:}

As oral health care providers, we can contribute to improvement of treatment outcomes by recognizing patients at risk and hence, the relevance of the present study. Being a university campus, majority of the subjects in the study belonged to higher socio - economic strata with higher levels of education and good oral hygiene levels associated with minimal gingival inflammation. Body mass index was therefore not significantly associated with periodontal disease. In light of the above, it may not be wrong to surmise that maintaining normal body weight with attention to adequate nutritional requirements may be important in reducing the risk of developing periodontal disease. Further, maintenance of adequate oral hygiene and normal body weight can reduce the overall inflammatory burden. Therefore, we may infer that absence of a state of chronic inflammation is a better predictor of periodontal health, as demonstrated by the significantly lower gingival index scores in periodontally healthy individuals.

\section{Acknowledgement:}

The authors would like to acknowledge the assistance provided by the auxiliary staff and postgraduates of the department of Periodontology, Manipal College of Dental Sciences, Manipal.

\section{Conflict of interest: Nil}

Funding: Nil

\section{References:}

1. Pi-Sunyer FX. Health implications of obesity. Am J Clin Nutr 1991;53:1595-603.

2. Thomas GN, Ho SY, Lam KS, et al. Cardiovascular Risk Factor Prevalence Study Steering Committee. Impact of obesity and body fat distribution on cardiovascular risk factors in Hong Kong Chinese. Obes Res 2004;12:180513.

3. Dalla Vecchia CF, Susin C, Rösing CK, et al. Overweight and obesity as risk indicators for periodontitis in adults. J Periodontol 2005;76:1721-8.

4. Al-Zahrani MS, Bissada NF, Borawskit EA. Obesity and periodontal disease in young, middle-aged, and older adults. J Periodontol 2003;74:610-5.

5. Alabdulkarim M, Bissada N, Al-Zahrani M, et al. Alveolar bone loss in obese subjects. J Int Acad Periodontol 2005; 7:34-8.

6. Saito T, Sashimazaki Y, Sakamoto M. Obesity and periodontitis. N Engl J Med 1998;339:482-3.

7. Beck J, Garcia R, Heiss G, et al. Periodontal disease and cardiovascular disease. J Periodontol 1996;67:1123-37. 
8. Albandar JM. Global risk factors and risk indicators for periodontal diseases. Periodontol 2002;29:177-206.

9. Silness J, Loe H. Periodontal disease in pregnancy. II. Correlation between oral hygiene and periodontal disease. Acta Odontol Scand 1964;22:121-35.

10. Loe H, Silness J. Periodontal disease in pregnancy. I. Prevalence and severity. Acta Odontol Scand 1963;21:533-51.

11. World Health Organization. Oral Health Surveys: Basic Methods, 4th ed. Geneva: World Health Organization 1997:6-39.

12. World Health Organization. Obesity: Preventing and Managing the Global Epidemic. Report of a WHO Consultation. World Health Organization Technical Report Series 894, i - xii, 1 - 253, 2000, World Health Organization, Geneva.

13. Kim EJ, Jin BH, Bae KH. Periodontitis and Obesity: A Study of the Fourth Korean National Health and Nutrition Examination Survey. J Periodontol 2011;82:533-42.
14. Ylostalo P, Suominen-Taipale L, Reunanen A, et al. Association between body weight and periodontal infection. J Clin Periodontol 2008;35:297-304.

15. WHO Expert Consultation. Appropriate body - mass index for Asian populations and its implications for policy and intervention strategies. Lancet 2004;363:15763.

16. Papapanou PN, Lindhe J, Sterrett JD, et al. Considerations on the contribution of ageing to loss of periodontal tissue support. J Clin Periodontol 1991;18:611-15.

17. Abdellatif HM, Burt BA. An epidemiological investigation into the relative importance of age and oral hygiene status as determinants of periodontitis. J Dent Res 1987;66:13-18.

18. $\mathrm{Xu} \mathrm{F}$, Yin $\mathrm{XM}$, Wang $\mathrm{Y}$. The association between amount of cigarettes smoked and overweight, central obesity among Chinese adults in Nanjing, China. Asia Pac J Clin Nutr 2007;16:240-7. 